\title{
La contribution de l'éducation à la performance de la croissance et du développement économique durable : Apport théorique et spécificité du cas marocain
}

\author{
Hanane LOUARDY ${ }^{\mathrm{a}^{*}} \&$ Abdelhak MOUSSAMIR ${ }^{\mathrm{a}}$ \\ ${ }^{a}$ Université Hassan II Casablanca, Maroc \\ * nina.louardy@gmail.com
}

\section{Résume}

L'objectif du papier est de revoir la contribution de l'éducation dans le processus d'une croissance économique durable et performante. Sur le plan méthodologique, une exploration théorique sera avancée en premier lieu pour évaluer l'importance de l'éducation dans le développement des économies et en deuxième lieu, les spécificités du système d'éducation marocain vont être relativement analysées pour en dédier les forces et les faiblesses. En dépit des avancements notables, le système d'éducation marocain souffre encore aujourd'hui du manque de la qualité et des performances supposées acquises dans le processus de scolarisation et d'éducation malgré sa contribution à l'amélioration de la performance économique confirmée dans la phase théorique.

Mots clés : développement durable, croissance inclusive, capital humain, éducation, enseignement de qualité, étude d'impact.

\section{The Contribution of education to the performance of growth and sustainable economic development: Theoretical contribution and specificity of the Moroccan case}

Abstract

The objective of the paper is to review the contribution of education in the process of sustainable and efficient economic growth. On the methodological level, a theoretical exploration will be advanced firstly to 
assess the importance of education in the development of economies and secondly, the specificities of the Moroccan education system will be relatively analyzed to dedicate the strengths and weaknesses. Despite notable progress, the Moroccan education system still suffers today from the lack of quality and performance acquired in the process of schooling and education despite its contribution to the improvement of economic performance confirmed in the theoretical phase.

Key words: sustainable development, inclusive growth, human capital, education, impact study.

\section{Introduction}

La croissance et le développement économique sont les soubassements stratégiques de toutes actions et politiques engagées par l'Etat dans le cadre du son processus de développement durable supposé instaurer une prospérité et une équité économique et sociale partagées entre toute la population d'un pays donné. Ce développement durable est dans la mesure d'être assuré par la dynamisation de plusieurs paramètres multidimensionnels dont une croissance inclusive et soutenue, qui demeure l'un des objectifs majeurs des Etats-nations. Le but étant de générer plus de richesses et de valeurs ajoutées en intégrant toutes catégories confondues de la population dans le processus de création des richesses, particulièrement dans un monde économique ouvert et global.

Ce nouveau paradigme dans les stratégies de développement est appuyé par l'essor et l'évolution d'une théorie économique et d'une pratique sociétale à l'échelle mondiale qui soutiennent et favorisent l'humain dans son environnement économique et social. Comme démontré par les théories de la pensée économique issues de l'approche endogène, le capital humain est une richesse immatérielle qui nécessite une vision et une attention particulière pour son développement et son amélioration durable et 
continue en vue d'exploiter ses fortes externalités sur la productivité totale des facteurs ainsi que sur la sphère sociale, politique et culturelle. Ce dernier s'avère être une variable centrale et une composante essentielle dans le mécanisme d'accumulation des richesses et des progrès technologiques (Grossman et Helpman, 1994 ${ }^{1}$ ).

Or, un individu opérationnel dans une économie doit tout d'abord être doté d'un revenu et des conditions de vie relativement convenables au sein de sa société (revenu minimum, accès aux services publics de base, logement décent, infrastructure de qualité et un cadre juridique et règlementaire adéquat à la bonne gouvernance). De ce fait, la scolarisation et l'éducation sont parmi les déterminants majeurs de la croissance économique (Mankiw et al. $1992^{2}$ ) et sont aussi les indicateurs les plus prometteurs et les plus performants en termes d'amélioration individuelle du niveau de vie et de participation active à la vie économique et donc, au développement collectif et global.

Ainsi, l'accès à la scolarisation et l'augmentation (ou la réduction) de la pauvreté peuvent être reliées par une causalité bidirectionnelle très réactive, étant donné que la pauvreté est le principal facteur de l'abondant scolaire, plus particulièrement des jeunes filles et notamment, dans les zones rurales ; tout en considérant que le marché de travail est une source importante de revenu pour la population globale, notamment la plus pauvre.

${ }^{1}$ GROSSMAN Gene and HELPMAN Elhanan, (1994). « Endogenous Innovation in the Theory of Growth ». Journal of Economic Perspectives, Volume 8, $\mathrm{N}^{\mathrm{O}}$. 1, pp. 23-44.

${ }^{2}$ MANKIW Gregory, ROMER David and WEIL David, (1992). « A Contribution to the Empirics of Economic Growth». The Quarterly Jounz4g of Economics, Volume 107, Issue 2, pp. 407437. 
A l'instar des autres pays en voie de développement et comme la problématique des inégalités sociales dont les inégalités d'accès à la scolarisation, à l'éducation et à la formation professionnelle présentent un phénomène international, le Maroc met l'emphase sur le développement d'une stratégie d'éducation et d'enseignement supérieur pour s'échapper à l'attrape du sous-développement, qui s'est accumulée de par l'histoire générant ainsi des situations de difficultés, de vulnérabilités et d'exclusion sociale de plus en plus accrues.

A cet effet, le modèle de la croissance économique du Maroc a centralisé l'individu au cœur d'une stratégie globale de développement et d'ouverture internationale commerciale et financière. De surcroît, un appel mondial à réagir face à l'inégalité sociale se consolide et subit récemment une intervention parallèle au niveau national de la part des autorités publiques chargées d'élaborer des programmes de lutte contre la pauvreté, la précarité et l'exclusion sociale et international de la part des institutions mondiales telles que les organismes de l'ONU dont la Banque Mondiale, FMI, PNUD et les ONG, qui sont très actives à ce niveau ${ }^{3}$.

Ces programmes et réformes à l'ordre du jour s'emploient à réduire la pauvreté dans sa dimension relative et absolue via des actions multidimensionnelles actives particulièrement sur le secteur de l'éducation et de l'enseignement marocain, qui assurent la continuité du processus de développement entamé et enchaîné par ces derniers.

${ }^{3}$ DUMONT Gérard-François, (2017). «QUELLES POLITIQUES CONTRE LA PAUVRETÉ ?» dans Stefano Zamagni, Marcelo Sanchez Sorondo. «Inclusive solidarity and integration of marginalized people», Libreri Editrice bscicana, pp.149-173 
Certes, l'accroissement des inégalités sociales pourrait être contrecarré par un accès favorable et obligatoire à la scolarisation et à l'éducation qui marque un investissement sur le long terme dans le capital humain de demain. En revanche, la redistribution efficiente des richesses est une tâche affectée à la responsabilité de l'Etat, qui doit mobiliser des ressources importantes pour établir des infrastructures appropriées et du personnel adéquat au milieu scolaire dans les deux zones urbaine et rurale. Des progrès remarquables (dont atteste le taux d'alphabétisation des marocains ${ }^{4}$ ) ont été réalisés mais des insuffisances demeurent et doivent être corrigées.

Par ailleurs, l'ouverture commerciale internationale du Maroc ne passe pas à l'abri des comptes car ses effets sont multiples sur l'économie nationale. L'engagement dans des politiques orientées à l'externe réduit les marges de manœuvre de l'Etat sur les secteurs sociaux et marginalise l'impact de ces secteurs sur le bien-être de la population notamment la tranche de la population la plus démunie.

Que nous rapporte la théorie sur la contribution de l'éducation à la performance de la croissance économique ? Et que représente cet apport théorique dans le modèle de développement économique du Maroc parallèlement à sa stratégie d'ouverture commerciale dynamique et intensive?

\section{I/ Revue de littérature}

La valeur ajoutée du capital humain qui s'affiche réellement dans sa contribution dynamique à la formation et à l'accumulation des richesses matérielles et immatérielles

\footnotetext{
${ }^{4}$ Rapport du Haut-Commissariat au Plan, (2018). « Les indicateurs sociaux du Maroc », Direction des statistiques, Edition 2018.
} 
a été sujet de vif débat et de profonde investigation dans plusieurs approches théoriques et empiriques et ce depuis la structuration de la pensée économique.

Autrement dit, la contribution de l'éducation à la création de la valeur ajoutée et dans la formation du PIB a été depuis longtemps analysée dans son angle économique le plus étroit malgré son impact conséquent sur le développement du capital humain, qui est source de croissance et de prospérité intergénérationnelle. Or, un accès élargie et en équité sociale qui garantit les mêmes opportunités pour toute la population fait récemment des échos, particulièrement dans les pays les moins avancés et les pays en développement. Ainsi, la diversité des analyses et des recherches à ce niveau ont démontrés le manque à gagner en cas d'une faiblesse d'investissement dans ce secteur et l'importance de la redistribution des revenus, qui devrait être assurée par les autorités publiques dans un cercle de valorisation du bien-être des populations les plus pauvres.

Les courants classique et néoclassique, souvent associé à la doctrine du «laissez-faire, laissez passer » ont misé sur l'importance du facteur travail sans se brancher et s'impliquer dans les mécanismes et les moyens par lesquels un travailleur devient qualifié pour réaliser ses tâches et acquérir un savoir-faire menant à une performance individuelle et donc à une productivité élevée, notamment avec les effets d'apprentissage et les effets d'expérience décortiqués dans les approches théoriques de la croissance endogène $e^{5}$.

${ }^{5}$ WEST Edwin George, (1964). «The role of education in nineteenth-century doctrines of political economy ». British Journal of Educational Studies, 12(2), pp. 161-172. 
Dans ce sens, Smith $(1776)^{6}$ explique dans son analyse des causes des richesses mondiales qu'il existe une forme et une approche de base dédiée à l'éducation et aux compétences développées par les individus sans se pencher sur l'explication de leurs origines et du processus de leurs formations. L'analyse à cet effet distingue l'individu de la seule fonction de consommation et lui attribue ainsi la fonction de productivité comme agent économique ${ }^{7}$. D'autres chercheurs tels que Marshall, Mill, Fisher ont confirmé l'approche de Smith qui stipule que la différence salariale est une différence du savoir-faire entre les personnes en mesure de travailler sans expliquer le processus de qualification nécessaire pour atteindre un seuil d'exigence et de valorisation dans les divers marchés de travail (West, 1964 ${ }^{8}$ ). Cependant, cette divergence marque les niveaux de développement des pays.

Alors que l'individu a été classé dans la catégorie du capital fixe car il coûte une dépense et génère en contrepartie un profit qui compense ses charges conçues pour sa survie et son travail même en termes d'éducation, de formation professionnelle et de migration car la vision classique considérée les travailleurs comme ayant des dotations égales, il est placé, depuis les années 50 et 60 dans la catégorie du capital immatérielle, en l'occurrence le capital humain.

${ }^{6}$ SMITH Adam, (1776). «Wealth of nations », Volume I, Book II, Editions of Cananique, pp. 264.

${ }^{7}$ SPENGLER Joseph, (1977). «Adam Smith on Human Capital », The American Economic Review, Volume 67, №. 1, Papers and Proceedings of the Eighty-ninth Annual Meeting of the American Economic Assocation (February, 1977), pp. 32-36.

${ }^{8}$ Ibidem. 
Ainsi, l'avènement d'une analyse basée sur l'utilité capitaliste a marqué que l'augmentation des richesses nationales est non seulement attribuée à l'accumulation des facteurs de production traditionnels, qui sont le capital, le travail, les terres et les ressources naturelles mais aussi attribuables dans un processus aux différentes externalités libérées par l'éducation sur le reste de l'économie, notamment sur l'accroissement de la performance de la production nationale.

L'équation de la formation du PIB a été enrichie par l'attribut et les retombées du capital humain et de l'éducation, introduites initialement par Schultz (1961a $)^{9}$ dans une fonction de production interne pour distinguer ses impacts et désigner son importance en tant que variable clé dans la contribution aux richesses nationales.

De surcroît, l'éducation s'est renouvelée de positionnement à présent pour s'acquérir une forme d'investissement rationnellement rentable (Walsh, 1935 ${ }^{10}$; Psacharopoulos, $1981^{11}$; Psacharopoulos, $1988^{12}$ ) dont les fruits sont à récolter dans le long terme et dont les impacts se profilent au niveau individuel, collectif, social et économique national et international. L'objectif dans cette exploration dans les courants théoriques a était de revoir les motifs derrière les dépenses d'investissement dans ce domaine précis d'éducation et de scolarisation en comparaison avec les autres domaines

${ }^{9}$ SCHULTZ Theodore, (1961). «Investment in Human Capital». The American Economic Review, Volume 51, №. 1, pp. 1-17.

${ }^{10}$ WALSH Robert, (1935). « Capital Concept Applied to Man». The Quarterly Journal of Economics, Volume 49, №. 2, pp. 255-285.

${ }^{11}$ PSACHAROPOULOS George, (1981). «Returns to Education: An Updated International Comparison ». Comparative Education, Volume 17 , No. 3, pp. 321-341.

${ }^{12}$ PSACHAROPOULOS George, (1988). «Education and Development ». The World Bank Research Observer, Volume 3(1), pp. 99-116. 
de production à but lucratif et rationnel comme la création des firmes, la production des produits pour la commercialisation et bien d'autres (Ben-Porath, $1967^{13}$ ).

La vision néoclassique de la croissance exogène a insisté sur l'importance de l'augmentation de la productivité totale des facteurs pour converger vers un état de développement avancé afin de dépasser l'état stationnaire auquel converge la quasitotalité des pays (Solow, 1956 $6^{14}$ ). Ainsi, la qualification des travailleurs et l'amélioration des capacités humaines par l'éducation et l'introduction des avancements scientifiques et technologiques ont entrainé un accroissement des revenus, des rendements et donc, des profits.

Dans cette perspective, les théories de la croissance endogène ${ }^{15}$ ont confirmé que l'éducation contribue au développement soutenable et à l'émergence d'un environnement économique stable et propice aux progrès économique et technologique. De surcroît, ces théories indiquent que le capital humain est en mesure de progresser en performance et d'améliorer sa productivité et sa compétence via l'effet d'apprentissage et d'expérience avancés dans un cadrage théorique dans lequel prime les politiques encourageant la convergence vers un état avancée par plusieurs canaux de croissance

13 BEN-PORATH Yoram, (1967). «The Production of Human Capital and the Life Cycle of Earnings ». Journal of Political Economy, Volume 75, N0 4, Part 1, pp. 352-365.

${ }^{14}$ SOLOW Robert, (1956). « A Contribution to the Theory of Economic Growth ». The Quarterly Journal of Economics, Volume 70, Nº 1 (Feb., 1956), The MIT Press, pp. 65-94.

15 HOWITT Peter, (2004). «Endogenous Grow55Productivity and Economic Policy: A ProgressReport». International Productivity Monitor, Centre for the Study of Living Standards, volume 8 , pp. 3-15. 
(Lucas, $1988^{16}$; Romer, $1990^{17}$; Helpman, 1992 ${ }^{18}$ ). Dont figurent essentiellement la technologie, l'innovation et l'évolution du savoir-faire et des nouvelles connaissances et techniques de production, qui sont appuyées au préalable par une scolarisation et une éducation appropriées assurant la qualification du capital humain, car ils sont considérés comme des indicateurs clés du développement des économies.

Par ailleurs, la capacité d'absorber les externalités de l'innovation technologique et de les adapter à un contexte et une situation spécifique à l'échelle interne nécessitent l'insertion dans un processus de réinvention animée par la qualification du capital humain (Rogers, $1983^{19}$ et $1987^{20}$ ).

La diffusion de la technologie implique sur le plan théorique deux hypothèses majeures qui se répercutent dans le pouvoir financier des parties engagées. Les canaux de diffusion de la technologie sont pratiquement soit issus des pays du Nord où les agents économiques (Etat, entreprises et population) s'inscrivent dans un processus d'investissement dynamique en recherche et innovation, qui exige un financement

${ }^{16}$ LUCAS Robert, (1988). « On the mechanics of economic development ». Journal of Monetary Economics Volume 22, Issue 1, pp. 3-42.

${ }^{17}$ ROMER Paul, (1990). «Endogenous Technological Change ». Journal of Political Economy Volume 98, $\mathrm{N}^{0} 5$, Part 2.

${ }^{18}$ HELPMAN Elhanan, (1992). «Endogenous Macroeconomic Growth Theory ». European Economic Review 36, pp. 237-267.

19 ROGERS Everett, (1983). «Diffusion of hgøvations». 3rd Edition, New York: The FreePress.

${ }^{20}$ ROGERS Everett, (1987). «The diffusion of innovations perspective ». In Neil Weinstein (Ed.), Taking Care: Understanding and Encouraging Self-Protective Behavior Cambridge. Cambridge University Press. pp. 79-94. 
considérable et, par conséquent, évitent la redondance qui s'avère être coûteuse par la communication des nouvelles idées (Rogers, $1983^{21}$ ). Soit, imposés aux pays du Sud, qui subissent ces progrès scientifiques par un transfert actif des nouvelles modes et tendances technologiques en optant à l'imitation qui stimule la réinvention et dans le long terme l'insertion dans les circuits technologiques (tel est le cas des pays émergents de l'Asie) et à l'utilisation (pour le reste des pays du Sud les moins avancés) (Kneller et al., $2009^{22}$ ).

La convergence est déterminée par une panoplie de variables clés y inclus la technologie (Schumpeter, 1934²3), l'éducation et les efforts fournis par les autorités en place pour stimuler le développement de ces derniers en termes d'investissement public concrétisé par des réformes internes et une ouverture à l'extérieur manifesté par des échanges commerciaux et des investissement étrangers ${ }^{24}$.

À présent, l'analyse de l'évolution économique dans son ensemble ne peut être complète que par l'étude de la contribution et des attributions de l'éducation et du

${ }^{21}$ Ibidem.

${ }^{22}$ KNELLER Richard, HENRY Michael and MILNER Chris, (2009). «Trade, technology transfer and national efficiency in developing countries ». European Economic Review, Volume 53, Issue 2, pp. 237-254.

${ }^{23}$ SCHUMPETER Joseph Alois, (1934). « The Theory of Economic Development: An Inquiry into Profits, Capital, Credit, Interest, and the Business Cycle ». Harvard Economic Studies 46.

${ }^{24}$ Jung Hong-Sang and Thorbecke Erik, (2003). «The impact of public education expenditure on human capital, growth, and poverty in Tanzania and Zambia: a general equilibrium approach ». Journal of Policy Modeling

Volume 25, Issue 8, pp. 701-725. 
capital humain dans la mesure où leurs impacts favorisent l'essor et le développement de l'innovation, la créativité, la technologie et les nouvelles idées.

On marque à ce niveau une différence de perception entre la scolarisation et l'éducation ${ }^{25}$. La scolarisation, étant particulièrement le sous-bassement de la branche totale de l'éducation et de l'évolution technologique, qui de son tour et après achèvement, est capable de former un individu pour une carrière professionnelle. Toutefois, la scolarisation, appuyée par l'Etat et les parents dans le cadre des communautés sociales, est assurée en obligation dans la quasi-totalité des pays avec une finalité principale, qui est de promouvoir les valeurs d'éducation et de former les citoyens de demain á l'échelle politique, économique et culturelle afin de les rendre également capable d'interagir avec les différentes institutions publiques et aussi dans la diversité des interactions sociales. Ainsi la scolarisation, à ce niveau, est loin de toute profitabilité, en revanche elle assume les préalables des progrès des nations.

Au-delà du secondaire, l'éducation devient de plus en plus coûteuse et amorce une spécialisation en vue de cibler un domaine de travail précis, d'où l'introduction des motifs économiques rationnels pour réaliser des profits. Les coûts et les dépenses réalisées dans le processus de l'éducation et de la formation professionnelle sont acquittés dans l'espoir d'une rémunération élevée et donc vers un compte rentable dans le futur, répercutant la valeur des compétences individuelles acquises par des personnes sur un marché de l'offre et de la demande.

Dans cette optique de pensée, l'individu acquis pendant ce cursus un ensemble de compétences et de performances utiles et nécessaires dans le cadre d'un travail, lui

${ }^{25}$ BREWER Dominic, MCEWAN Patrick, (2010). «Economics of Education ». 1st Edition, Elsevier. 
attribuant un avantage compétitif, qu'il convient de valoriser par des formations et des expériences multiples sur le marché de travail. De même, l'effort et l'énergie dédiés à l'apprentissage et à la qualification par une personne pendant son éducation doivent, tout de même, être remboursés.

Partant de cet enchaînement d'idée, l'éducation, en vue de former un capital humain performant et compétent qui se dote d'une valeur économique, est soumise aux influences des autres formes du capital en tant qu'investissement rentable et rationnel pour un profit futur. L'équilibre du marché par le principe de l'offre et de la demande ainsi que la libre circulation des capitaux et des personnes vers les zones les plus productives considèrent l'investissement dans l'éducation comme un engagement dans le but de profiter des rendements croissants débloqués par ce dernier. Les personnes, les familles et les Etats investissent dans les systèmes d'éducation performants dans le but d'améliorer le bien-être individuel et de la communauté dans son ensemble. Ceci explique en grande partie l'avancement technologique, scientifique et économique des pays du Nord, qui mobilisent des capitaux colossaux à ce secteur stratégique.

\section{II/ Analyse empirique du cas du Maroc}

Le Maroc, pays en développement classé par la Banque Mondiale dans la classe des économies à revenu intermédiaire tranche inférieure, dispose d'un système éducationnel qui se caractérise par un héritage de la période coloniale, d'où l'ancrage sur la langue française comme principale langue secondaire et langue des affaires, ainsi que des coutumes et des traditions qui renforcent le rôle de la langue arabe et des valeurs 
islamiques dans les compositions linguistiques et culturelles de ce dernier ${ }^{26}$.

En revanche, l'avènement d'un marché international global, notamment avec l'évolution des nouvelles technologies de communication et avec l'ouverture de plus en plus croissante des économies et des nations, les systèmes éducatifs construits sur le long terme, subissent à présent des comparaisons internationales et des politiques d'appui hétérogènes (conduites par les institutions internationales) dans le but de réaliser des plus-values et des retombées positives sur le reste de l'économie, permettant plus particulièrement aux pays du Sud de rattraper le train du développement économique et technologique des pays du Nord raté il y a plusieurs décennies.

Á cet effet et à l'instar des pays en développement, le système éducationnel au Maroc affronte un ensemble de défis et de carences historiques incorporant un certain nombre de contradictions et d'expériences négligées dans le cadre du développement économique et du creusement des inégalités sociales au sein du territoire marocain, qui se manifestent parallèlement au processus de transition économique.

L'économie du Maroc qui peine à s'adapter à ce système mondial globalisé par un avancement industriel et technologique notable typiquement à une panoplie de pays en développement ${ }^{27}$, a-t-elle réussi à adapter et développer un système éducatif qui répond aux nouvelles exigences du progrès national et mondial ?

${ }^{26}$ SALMI Jamil, (1987). «Language and schooling in Morocco». International Journal of Educational Development, Volume 7, Issue 1, pp. 21-31.

${ }^{27}$ HARRIGAN Jane and EL-SAID Hamed, (2010). « The Economic Impact of IMF and World Bank Programs in the Middle East and North Africa: A Case Study of Jordan, Egypt, Morocco and Tunisia, $1983-2004$ ». Review of Middle East Economics and Finance, Volume 6, Issue 2, pp. 1-25. 
Après plusieurs décennies de politiques autocentrées entraînant une crise d'endettement et un déséquilibre macroéconomique flagrant, les années 80 ont marqué l'engagement du Maroc dans des réformes et des mesures d'ajustement structurel, imposant une politique extérieur d'ouverture commerciale progressive et une réduction des dépenses de publiques dans la sphère des secteurs sociaux. À cette époque, le système d'éducation du Maroc se caractérisait par un analphabétisme et un illettrisme élevés de la population ${ }^{28}$ et une infrastructure de base faiblement établie dans le territoire, causant une inégalité d'accès des différentes tranches de la population à ce service public de base, tout particulièrement la scolarisation des enfants dans les zones enclavées $^{29}$.

De ce fait, une réforme globale, basée sur la qualité pour promouvoir les secteurs économiques et fondée sur l'égalité des chances plaidant pour la prospérité et l'équité sociale, s'est imposée. La réforme de l'éducation a affronté une réalité économique et politique bien différente et a nécessité un budget lourd qui assure, d'abord la stabilité et, dans ce qui suit, une transition progressive réelle, particulièrement après la dégradation du système social dans son ensemble, comme

${ }^{28}$ LAVY Victor, and SPRATT Jennie, (1997). «Patterns of Incidence and Change in Moroccan Literacy ». Focus on Adult Skills, Comparative Education Review, Volume 41, N ${ }^{0} .2$, pp. $120-141$.

${ }^{29}$ CHEDATI Brahim, (2005). «Les cycles d'enseignement primaire, secondaire collégial et qualifiant, Quelle efficacité ? Quelle équité ? À quels coûts ?», pp. 109-153. http://www.ires.ma/ 
résultat des mesures du programme d'ajustement et le manque d'une vision de long terme ${ }^{30}$.

Par ailleurs, la complexité qui régit les interactions entre les différentes composantes et déterminants de la croissance et des performances économiques dont l'éducation se procure une place primordiale, incite à mettre en exergue l'importance de l'accumulation du capital dans le développement et les progrès technologique des nations. Ainsi, le rapport entre un niveau de vie décent et un niveau d'éducation important est généralement apprécié pour l'impact bidirectionnel que ces deux variables exercent entre eux tout en impliquant des taux de croissance élevés ${ }^{31}$.

Graphique 1 : Evolution des dépenses de consommation des ménages marocains et du PIB par habitant du Maroc entre 1990-2017

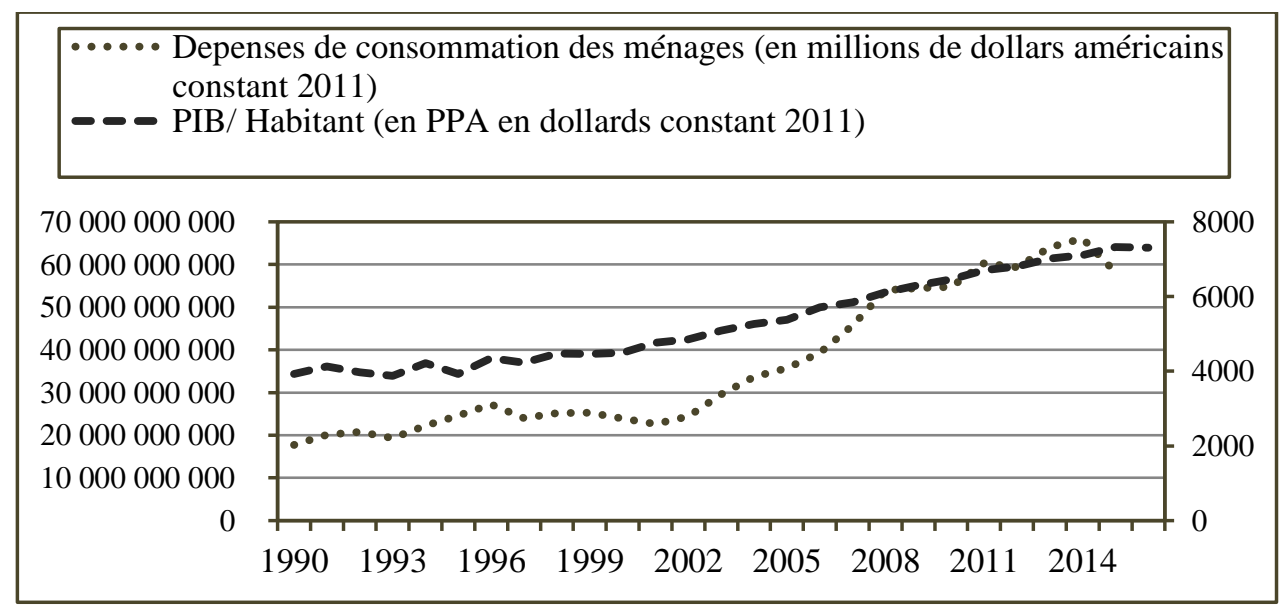

${ }^{30}$ SEDDON David, (1989). « The Politics of Adjustment in Morocco », Structural Adjustment in Africa. Part of the International Political Economy Series book series (IPES), B. K. Campbell et al. (eds.), pp. 234-265.

${ }^{31}$ HADIR Adnane and LAHRECH Abdelmounaim, (2015). « Human Capital Development and Economic Growth in Morocco». Ifterextional Journal of Education and Human Developments, Volume 1 No. 1, pp. 49-73. 
Source : Données du HCP et de la Banque Mondiale.

Ces dernières décennies, le Maroc a réalisé une évolution relative en termes de richesse moyenne par habitant assurant des dépenses de consommation élevées par ménages et par conséquent un choix en dépense d'éducation bien élargi (école privée, équipements et fournitures, formations et accompagnements et bien d'autres). Au Maroc, l'éducation est assurée en majeur partie comme un service public par l'Etat avec une tendance de privatisation de plus en plus accrue, illustrée relativement par une part de 14\% dans le segment de la scolarisation primaire gérée par le secteur privée en 2016 ainsi qu'une part de l'enseignement supérieur privatisée qui ne cesse d'augmenter ${ }^{32}$.

Les caractéristiques démographiques de la population dans le cas du Maroc constituent un atout et un avantage au développement économique, car avec un taux de croissance annuel démographique de $1 \%$ entre 2012 et 2030 (contre 1,3\% entre 1990 et $2012)^{33}$ et un taux de population jeune et active (entre 18 et 59) capable d'accéder au marché de travail, qui est aux alentours de $62,8 \%$ en $2017^{34}$, le capital humain du Maroc est porteur d'espoir. De surcroît, une culture d'ouverture sur l'externe et sur les différentes cultures mondiales constituent un déterminent majeur à la diversification des valeurs éducationnelles et des compétences requises pour le développement interne. En

32 EZZRARI Abdeljaouad, (2018). «Dépenses publiques et dépenses des ménages en éducation », Observatoire des conditions de vie de la population, les Brefs du Plan $\mathrm{N}^{\circ} 4, \mathrm{HCP}$.

${ }^{33}$ https://www.unicef.org/french/infobycountry/morocco_statistics.html

34 Rapport du Haut-Commissariat au Plán, $2(6918)$. «Les indicateurs sociaux du Maroc»,

Direction de la statistique, Edition 2018. 
atteste le nombre croissant des étudiants marocains à l'étranger et dans le sens inverse, l'accroissement des étudiants étrangers au $\operatorname{Maroc}^{35}$.

Ce potentiel façonne le capital humain du pays qui est susceptible de relancer l'activité économique en performance sous réserve qu'il s'attache à un développement considérable et à une qualification réelle appuyés simultanément par les autorités publiques et privées du pays par un investissement physique et immatériel (Barro, $2000^{36}, 2001^{37}$ et $2013^{38}$ ). L'amélioration de la qualité du capital humain renforce, à la fois, ses capacités productives et son aptitude d'absorption des nouvelles technologies et d'adaptation aux nouvelles contraintes des progrès internationaux qui s'avèrent être reconsidérées afin de bénéficier du stock mondial d'innovations (Lucas, 1990 39).

L'éducation est dans la mesure de projeter l'individu dans le monde de travail professionnel pour se procurer un revenu et donc pour s'échapper de la pauvreté intergénérationnelle et absolue, particulièrement pour les classes sociales les plus démunies. Toutefois et pour l'importance du secteur de l'éducation et la forte corrélation entre le budget alloué à son financement et la croissance économique, notamment avec l'existence des institutions de qualité et de la bonne gouvernance au

35 Ministère de l'éducation nationale, de la Formation Professionnelle, de l'Enseignement Supérieur et de la Recherche Scientifique. https://www.enssup.gov.ma/fr/

${ }^{36}$ BARRO Robert, (2000). « Education and Economic Growth ». Paris : OECD.

37 BARRO Robert, (2001). «Human Capital and Growth». American Economic Review, American Economic Association, volume 91(2), pp. 12-17.

38 BARRO Robert, (2013). «Education afd 26epnomic Growth ». Annals of Economics and Finance, Society for AEF, volume 14(2), pp. 301-328.

39 LUCAS Robert, (1990). «Why doesn't capital flow from rich to poor countries? », The American Economic Review, 80(2), pp. 92-96. 
sein de ces dernières (Acemoglu et al., 2006 ${ }^{40}$; Blankenau et al., 2007 41 ), cet investissement ne peut contribuer à la dynamisation du secteur et à l'expansion de ses externalités aux autres secteurs stratégiques de production que sur le long terme, particulièrement avec l'évolution démographique et la transition économique structurelle des pays en développement comme le Maroc.

Graphique 2 : Evolution des dépenses publiques totales dans le secteur de l'éducation (\% du PIB) au Maroc entre 1980 et 2015

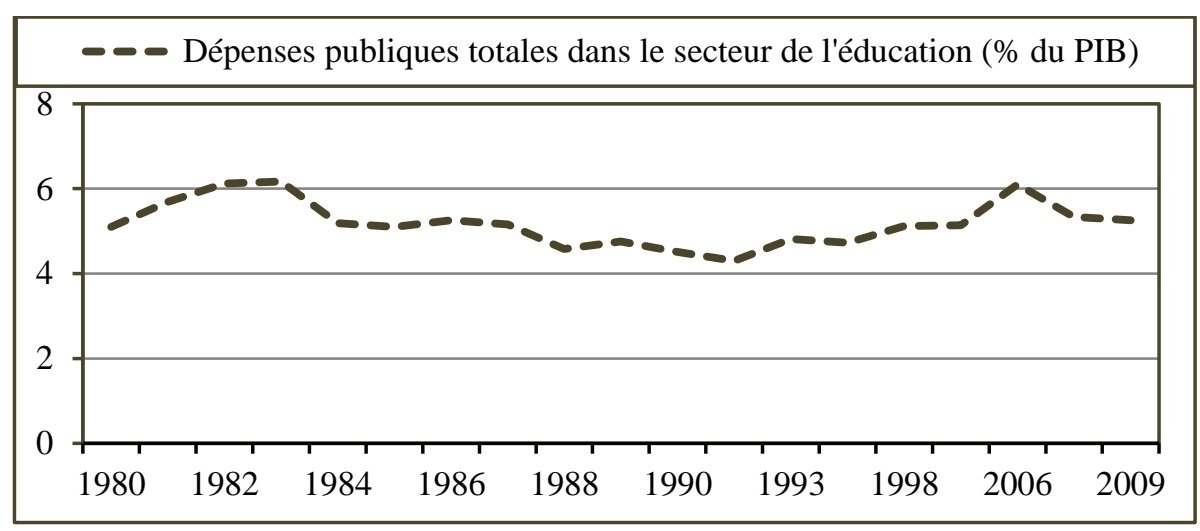

Source : Données de la Banque Mondiale.

Le budget consacré à un secteur stratégique tel que celui de l'éducation évolue sur une courbe de stabilité relativement constante, qui ne dépasse pas dans la rigueur $6 \%$ du PIB (tout en prenant en considération l'évolution même du PIB), avec près de $80 \%{ }^{42}$ consacrée aux dépenses de fonctionnement de base, ne reflète pas l'importance

${ }^{40}$ ACEMOGLU D, AGHION P, ZILIBOTTI F., (2006). « Distance to frontier, selection, and economic growth », Journal of the European Economic association, 4(1), pp. 37-74.

41 BLANKENAU W, SIMPSON N, TOMLJANOVICH M., (2007). «Public education expenditures, taxation, and growth: Linking data to theory », The American economic review, 97(2), pp. 393-397. $\quad$ ( 265

${ }^{42}$ Ministère de l'éducation nationale, de la Formation Professionnelle, de l'Enseignement Supérieur et de la Recherche Scientifique. https://www.enssup.gov.ma/fr/ 
de ce secteur dans l'ensemble. En d'autres termes, ce budget prend en considération l'élargissement de l'effectif des élèves, des professeurs et des cadres professionnels du secteur, qui est financé majoritairement par des fonds publics. Les dépenses publiques ont été réajustées ces dernières années dans l'espérance de marquer la qualité de l'éducation et de l'enseignement.

Dans ce cadre de pensée et malgré la diversification des initiatives, le système éducatif marocain souffre d'un ensemble de déséquilibres fondamentaux dont l'inadéquation des choix et le manque de la flexibilité ainsi que l'inadaptation relative avec les développements récents du monde externe et du marché de travail national ${ }^{43}$. Les réformes annoncées et appliquées pour relancer le système éducatif au Maroc avaient parmi les objectifs primordiaux l'insertion d'une vision mondialisée dans ses dispositifs et d'y incorporer la stratégie et le modèle du développement marocain pour accompagner son ouverture et sa transition économique structurelle en valorisant les spécificités propres au pays et en exploitant le potentiel de son capital humain ${ }^{44}$.

En revanche, l'ouverture économique, financière et commerciale dans laquelle s’est engagée le Maroc a rétrécis le champ d'actions dont opère le gouvernement

${ }^{43}$ DIYEN Hayat, (2004). «Reform of Secondary Education in Morocco: Challenges and Prospects », PROSPECTS, 34(2), pp. 211-222.

${ }^{44}$ IFA Adel and GUETAT Imène, (2018). « Does public expenditure on education promote Tunisian and Moroccan GDP per capita? A 2 SO approach ». The Journal of Finance and Data Science, Volume 4, Issue 4, pp. 234-246. 
(Barro, $1990^{45}$ et $1991^{46}$; Rodrik, $1998 b^{47}$ ) en défaveur des secteurs sociaux dont l'éducation et le développement de l'innovation et des activités de recherche et développement occupent une place centrale. En atteste le budget consacré par les décideurs publics au Maroc aux secteurs de haute technologie et aux activités de recherche et développement qui ne dépasse pas $1 \%$ (0,8\% du PIB en 2017) depuis plusieurs années.

Tableau 1 : Dépenses en recherche et développement (en \%du PIB)

\begin{tabular}{|l|l|l|l|}
\hline Année & Maroc & $\begin{array}{l}\text { Moyenne des pays à } \\
\text { revenu } \\
\text { intermédiaire }\end{array}$ & $\begin{array}{l}\text { Moyenne } \\
\text { mondiale }\end{array}$ \\
\hline 2000 & $0,61 \%$ & $0,64 \%$ & $2,06 \%$ \\
\hline 2017 & $\begin{array}{l}0,80 \%(0,34 \% \text { en } \\
2016)\end{array}$ & $1,48 \%$ & $2,30 \%$ \\
\hline
\end{tabular}

Source : Données de la Banque Mondiale.

Les actions entretenues et les efforts concentrés dans le cadre d'une stratégie globale ont marqué l'évolution de plusieurs indicateurs clés dans la progression du

${ }^{45}$ BARRO Robert, (1991). « Economic growth in cross-section of countries ». Quarterly Journal of Economics, 106(2). pp. 407-443.

${ }^{46}$ BARRO Robert, (1990). « Government Spending in a Simple model of Endogenous Growth». Journal of Political Economy, Vol. 98, No. 5, Part 2: The Problem of Development: A Conference of the Institute for the Study (of $26 \%$ e Enterprise Systems, pp. S103-S125.

${ }^{47}$ RODRIK Dani, (1998). « Why do more open economies have bigger governments? ». Journal of Political Economy 106, pp. 997-1032. 
système éducatif marocain ${ }^{48}$. Parmi d'autres, le taux d'alphabétisation, le taux d'abondement scolaire, le taux d'achèvement scolaire ont été améliorés concrètement avec l'évolution des effectifs. Le nombre des étudiants inscrits et ayant achevés un cycle d'éducation, allant du primaire au secondaire collégial et qualifiant public, a marqué un accroissement réel dû entre autres à des investissements physiques importants dans ce secteur et à la valeur attribué à l'éducation dans notre société comme élément clé du développement personnel et professionnel (rendement économique et social important).

Á cet effet, l'effectif des professeurs et le nombre des écoles suivent également la tendance et ont en conséquence accusé un accroissement prééminent pour s'établir et s'installer relativement dans la plupart des régions du territoire avec des concentrations plus au moins intenses dans les grandes villes, notamment l'axe Casablanca et Rabat.

Tableau 2 : Taux net de scolarisation par niveau d'éducation

\begin{tabular}{|l|l|l|l|l|}
\hline $\begin{array}{l}\text { Taux net de } \\
\text { scolarisation }\end{array}$ & Primaire & $\begin{array}{l}\text { Secondaire } \\
\text { collégial }\end{array}$ & $\begin{array}{l}\text { Secondaire } \\
\text { qualifiant }\end{array}$ & Supérieur \\
\hline $2014 / 2015$ & $98.27 \%$ & $87,6 \%$ & $61,1 \%$ & 33.72 (en 2016) \\
\hline $2008 / 2009$ & $92.94 \%$ & 52,3 & 32,3 & 11 (en 2007) \\
\hline $2000 / 2001$ & $70.67 \%$ & $55.99 \%$ & 29,7 & --- \\
\hline
\end{tabular}

Source : Données du HCP et du ministère de l'éducation nationale.

Sur le plan quantitatif et depuis l'indépendance du pays, les projets d'appui pour renforcer la structure du secteur éducatif au Maroc ont connu une prolifération pour accompagner la transition démographique et économique. En revanche, la répartition de

${ }^{48}$ IFA Adel and GUETAT Imène, (2018). op. cit. 
l’infrastructure de base (cités, cantines, internats), écoles (dont celles su secteur privé) et établissements d'éducation appartenant à ce secteur est inégalement répartie dans le territoire du Maroc et concentrée généralement dans les grandes villes.

Le caractère inégal d'accès à la scolarisation et à l'éducation entre les villes et entre les zones urbaines et rurales nuisent au développement du secteur et par voie de conséquence au progrès de l'économie dans son ensemble. Les systèmes régionaux d'éducation au Maroc dissimulent un caractère d'inégalité territoriale avec une inégalité de genre qui impacte généralement les filles dans les zones rurales, particulièrement les zones les plus enclavées. L'absence d'accompagnement pour cette tranche des élèves et l'inégalité et la disparité des infrastructures de base et des équipements dans les établissements d'éducation (primaire, secondaire et lycée) ainsi qu'une culture privilégiant l'investissement dans l'éducation des garçons (aptitudes négatives des parents) handicapent le développement économique et social et défavorisent l'évolution du niveau de vie et du bien-être de la population ${ }^{49}$.

Graphique 3 : Inégalité-Distribution des revenus au Maroc

49 EL ALAOUİ Aicha, (2016). «Impact of women's education on the economic growth: An empirical analysis applied to Morocco, Algeria, Tunisia, and Egypt ». International Journal of Social Sciences and Education Research, Votome 2, Issue 3, pp. 960-979. 


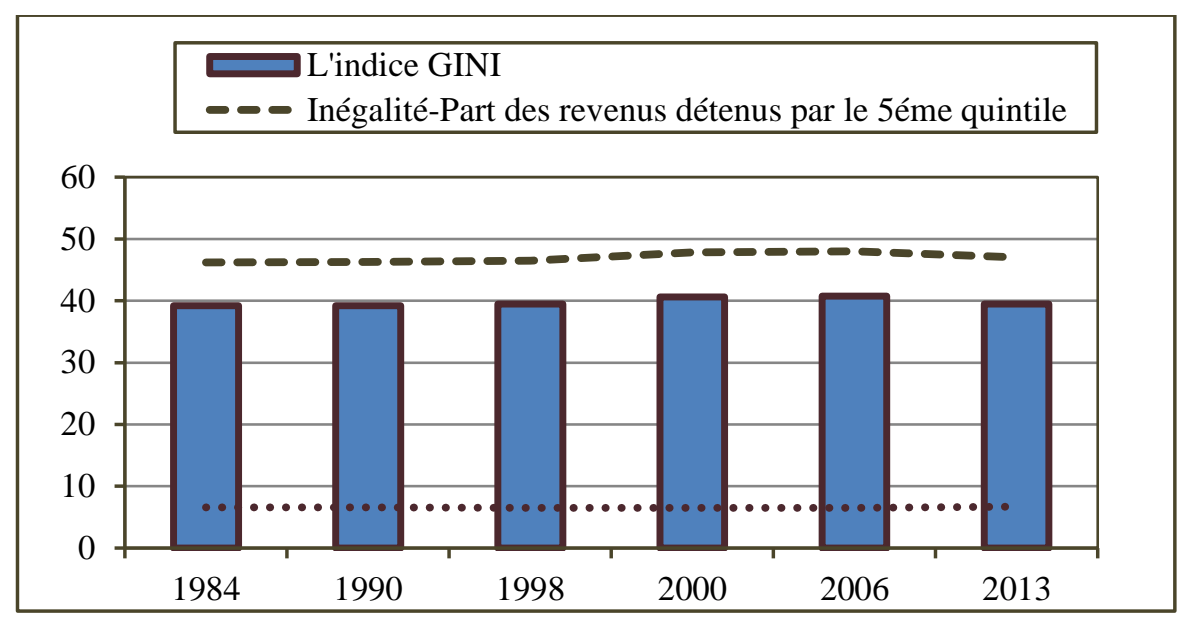

Source : Données du HCP.

La pauvreté et le renchérissement du coût de la vie, l'instabilité des foyers familiaux et les valeurs attribuées à la scolarisation dans des régions différentes du pays sont parmi les principales causes de l'abondant scolaire au Maroc et dans la plupart des pays du Sud, plus précisément dans la phase du collégial et du collégial qualifiant ${ }^{50}$.

Le succès réalisé sur le plan quantitatif, a il marqué la qualité créée pendant ce processus d'éducation ? Que rapportent ces chiffres aberrants sur le rendement du capital humain formé pour affronter les défis du développement et du progrès économique au Maroc? Les programmes et les mesures des réformes appuyées au Maroc ont misé principalement sur la quantité, renforçant le taux d'accès à la scolarisation pour généraliser l'alphabétisme, au détriment de la qualité d'éducation et d'enseignement, qui, pour plusieurs analystes, a connu une très forte dégradation ${ }^{51}$. Les compétences acquises (même en apprentissage de base) par les élèves $\backslash$ étudiants sont relativement affaiblies par l'inadéquation du programme, qui n'est pas dans la mesure

${ }^{50}$ CHEDATI Brahim, (2005). op. cit

51 TAWIL Sobhi, CERBELlE Sophie, AMAPOLA Alama, (2010). «Education au Maroc: analyse du secteur ». Documents de l'UNESCO N 48223, Bureau multipays pour le Maghreb, pp. 01-90. 
de relancer les progrès attendus créant ainsi un écart significatif entre le niveau réel et les compétences requises pour l'atteindre ${ }^{52}$. La productivité scientifique nationale s'établie à près de $0,1 \%$ de la production mondiale en $2017^{53}$.

Figure 1 : Niveau et performances en lecture, mathématiques et sciences acquises à l'école marocaine (élèves de 15 ans)

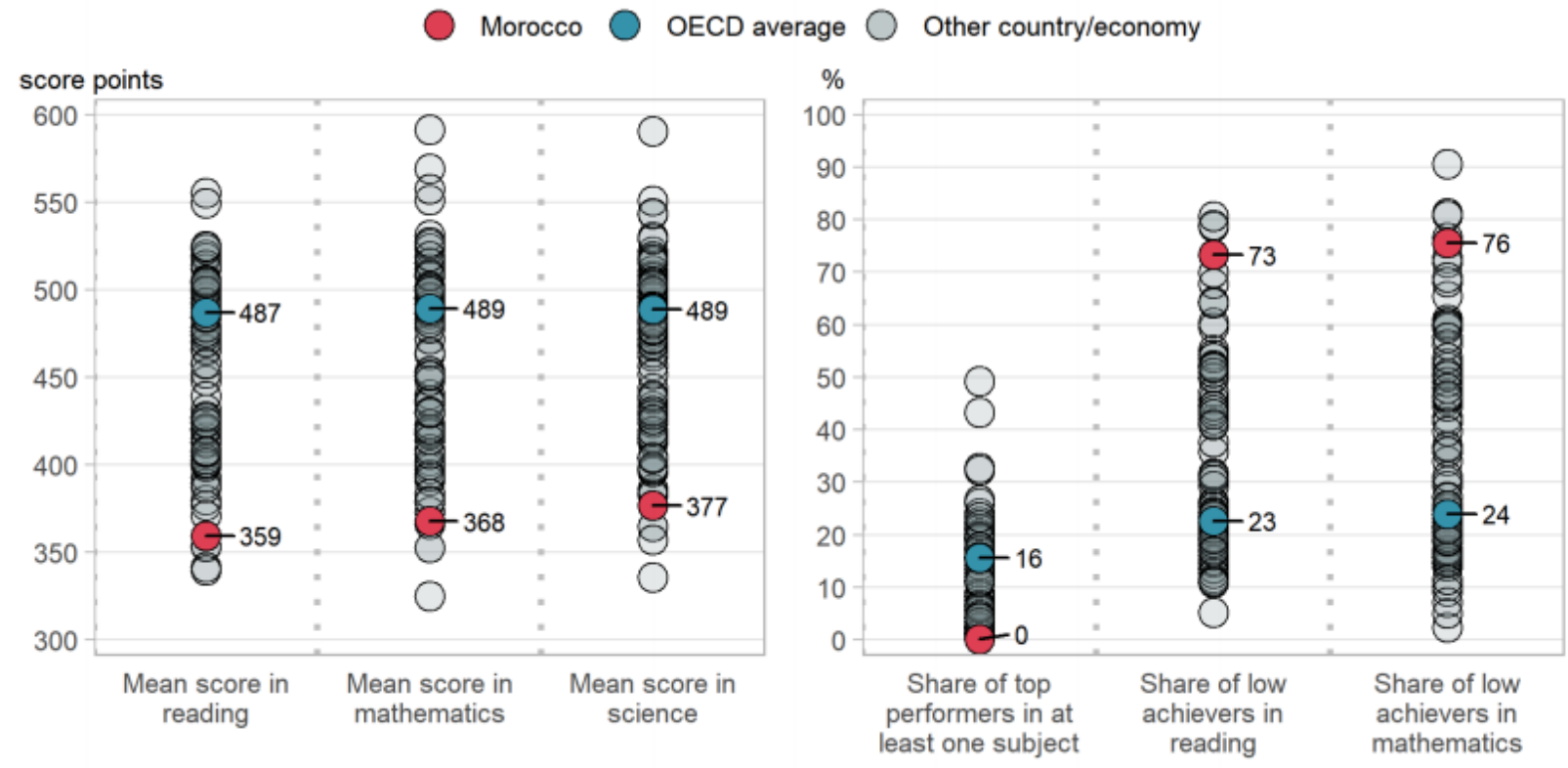

Source : OCDE, Program for International Student Assessment (PISA), Données 2018.

Les difficultés d'apprentissage dans l'éducation qu'affrontent les élèves font référence à la qualité de la scolarisation et à la qualité des programmes entretenus dans cette branche (figure 1). Toutefois le statut socio-économique des familles et des parents des élèves jouent un rôle crucial dans la performance de ces derniers, car les dépenses de consommation, en santé et en accompagnement pour l'éducation sont plus élevées ${ }^{54}$.

${ }^{52}$ OECD report, (2018). « Program for International Student Assessment (PISA): results from PISA 2018». Country note 2018.

\section{${ }^{53}$ https://www.inforisk.ma/}

${ }^{54}$ OECD report, (2018). 
Il est important de mentionner que la qualité de la prestation attribuée par le secteur de l'éducation est fortement corrélée avec la tendance de la croissance économique pour ses effets positifs ${ }^{55}$. A vrai dire, l'amélioration de la qualité de l'éducation au Maroc est supposée impacter la productivité et le rendement du travail et des richesses humaines au sein de l'économie.

\section{Conclusion}

L'éducation se procure à présent une place centrale dans le développement des pays et des économies pour son impact sur l'amélioration de la productivité et sur la progression technologique et l'essor des nouvelles idées ainsi que l'amélioration du bien-être individuel et de la société dans son ensemble.

Dans le cadre théorique, le courant classique a relié l'éducation avec la citoyenneté, le respect des lois et des règles, la prospérité économique, sociale et culturelle. L'avènement de la notion de l'utilitarisme a marqué l'introduction de l'éducation dans la sphère des investissements rationnellement rentables dont les impacts sont à apprécier dans le long terme. Toutefois les approches modernes de la pensée économique ont centralisé la scolarisation, l'éducation et les activités de recherche et développement dans les stratégies de développement en tant que variables réactives à l'évolution des progrès économiques.

${ }^{55}$ GLEWWE Paul, MAÏGA Eugénie and HAOCHI Zheng, (2014). «The Contribution of Education to Economic Growth: A Review of the Evidence, with Special Attention and an Application to Sub-Saharan Africa ». World Development, Volume 59, pp. 379-393. 
$\mathrm{Au}$ Maroc, les ressources humaines requièrent une importance cruciale sur le plan quantitatif et qualitatif. Ainsi, le nombre des personnes actifs sur le marché de travail, le nombre des élèves, le nombre des professeurs et des cadres au niveau administratif, le nombre des écoles et des établissements (destinés à la scolarisation, éducation, enseignement et formation professionnelle) sont en accroissement continu, absorbant de plus en plus d'effectif dans une finalité ultime de consolider le capital humain et affronter les défis socio-économiques ainsi que d'instaurer un environnement propice à la croissance économique valorisant l'investissement dans les personnes.

Les dépenses en termes d'éducation sont diversifiées et impactent plusieurs aspects de la vie quotidienne des personnes concernées, qui forment le capital humain de demain. Parmi d'autres, les dépenses d'enseignement organisé par niveau (primaire, secondaire, secondaire qualifiant, supérieur), les dépenses pour garantir des bonnes conditions de vie et de santé, les formations professionnelles ou complémentaires en cours de travail et d'éducation et, en dernier lieu, les dépenses de migration aménagées pour profiter des opportunités de travail et d'éducation.

Pour le rendement économique et social que procure l'éducation, l'équité et l'égalité d'accès à ce service de base doivent être assurées en obligation en réservant à ce secteur stratégique des taux d'investissement élevés, stimulant la transition vers un état de développement considérable et améliorant la qualité qui demeure à nos jours relativement dégradée.

\section{Références bibliographiques}

ACEMOGLU D, AGHION P, ZILIBOTTI F., (2006). « Distance to frontier, selection, and economic growth », Journal of the European Economic association, 4(1), pp. $37-74$.

BARRO Robert, (1990). « Government Spending in a Simple model of Endogenous Growth». Journal of Political Economy, Vol. 98, No. 5, Part 2: The Problem of 
Development: A Conference of the Institute for the Study of Free Enterprise Systems, pp. S103-S125.

BARRO Robert, (1991). «Economic growth in cross-section of countries ». Quarterly Journal of Economics, 106(2). pp. 407-443.

BARRO Robert, (2000). «Education and Economic Growth». Paris : OECD.

BARRO Robert, (2001). «Human Capital and Growth ». American Economic Review, American Economic Association, volume 91(2), pp. 12-17.

BARRO Robert, (2013). «Education and Economic Growth». Annals of Economics and Finance, Society for AEF, volume 14(2), pp. 301-328.

BEN-PORATH Yoram, (1967). «The Production of Human Capital and the Life Cycle of Earnings ». Journal of Political Economy, Volume 75, N0 4, Part 1, pp. 352365.

BLANKENAU W, SIMPSON N, TOMLJANOVICH M., (2007). «Public education expenditures, taxation, and growth: Linking data to theory », The American economic review, 97(2), pp. 393-397.

BREWER Dominic, MCEWAN Patrick, (2010). «Economics of Education ». 1st Edition, Elsevier.

CHEDATI Brahim, (2005). « Les cycles d'enseignement primaire, secondaire collégial et qualifiant, Quelle efficacité ? Quelle équité ? À quels coûts ? », pp. 109-153. http://www.ires.ma/

DIYEN Hayat, (2004). « Reform of Secondary Education in Morocco: Challenges and Prospects », PROSPECTS, 34(2), pp. 211-222.

DUMONT Gérard-François, (2017). «QUELLES POLITIQUES CONTRE LA PAUVRETÉ ? » dans Stefano Zamagni, Marcelo Sanchez Sorondo. «Inclusive solidarity and integration of marginalized people », Libreri Editrice Vaticana, pp.149-173.

EL ALAOUİ Aicha, (2016). « Impact of women's education on the economic growth: An empirical analysis applied to Morocco, Algeria, Tunisia, and Egypt ». International Journal of Social Sciences and Education Research, Volume 2, Issue 3, pp. $960-979$.

EZZRARI Abdeljaouad, (2018). «Dépenses publiques et dépenses des ménages en éducation », Observatoire des conditions de vie de la population, les Brefs du Plan $\mathrm{N}^{\circ} 4$, HCP. 
GLEWWE Paul, MAÏGA Eugénie and HAOCHI Zheng, (2014). « The Contribution of Education to Economic Growth: A Review of the Evidence, with Special Attention and an Application to Sub-Saharan Africa ». World Development, Volume 59, pp. 379-393.

GROSSMAN Gene and HELPMAN Elhanan, (1994). «Endogenous Innovation in the Theory of Growth ». Journal of Economic Perspectives, Volume 8, NO. 1, pp. $23-44$.

HADIR Adnane and LAHRECH Abdelmounaim, (2015). «Human Capital Development and Economic Growth in Morocco ». International Journal of Education and Human Developments, Volume 1 No. 1, pp. 49-73.

HARRIGAN Jane and EL-SAID Hamed, (2010). « The Economic Impact of IMF and World Bank Programs in the Middle East and North Africa: A Case Study of Jordan, Egypt, Morocco and Tunisia, 1983 - 2004 ». Review of Middle East Economics and Finance, Volume 6, Issue 2, pp. 1-25.

HELPMAN Elhanan, (1992). «Endogenous Macroeconomic Growth Theory ». European Economic Review 36, pp. 237-267.

HOWITT Peter, (2004). «Endogenous Growth, Productivity and Economic Policy: A Progress Report ». International Productivity Monitor, Centre for the Study of Living Standards, volume 8, pp. 3-15.

IFA Adel and GUETAT Imène, (2018). « Does public expenditure on education promote Tunisian and Moroccan GDP per capita? ARDL approach ». The Journal of Finance and Data Science, Volume 4, Issue 4, pp. 234-246.

Jung Hong-Sang and Thorbecke Erik, (2003). « The impact of public education expenditure on human capital, growth, and poverty in Tanzania and Zambia: a general equilibrium approach ». Journal of Policy Modeling, Volume 25, Issue 8, pp. 701-725.

KNELLER Richard, HENRY Michael and MILNER Chris, (2009). «Trade, technology transfer and national efficiency in developing countries ». European Economic Review, Volume 53, Issue 2, pp. 237-254.

LAVY Victor, and SPRATT Jennie, (1997). «Patterns of Incidence and Change in Moroccan Literacy ». Focus on Adult Skills, Comparative Education Review, Volume 41, N0. 2, pp. 120-141.

LUCAS Robert, (1988). «On the mechanics of economic development ». Journal of Monetary Economics Volume 22, Issue 1, pp. 3-42. 
LUCAS Robert, (1990). «Why doesn't capital flow from rich to poor countries? », The American Economic Review, 80(2), pp. 92-96.

MANKIW Gregory, ROMER David and WEIL David, (1992). «A Contribution to the Empirics of Economic Growth ». The Quarterly Journal of Economics, Volume 107, Issue 2, pp. 407-437.

OECD report, (2018). « Program for International Student Assessment (PISA): results from PISA 2018». Country note 2018.

PSACHAROPOULOS George, (1981). «Returns to Education: An Updated International Comparison ». Comparative Education, Volume 17, No. 3, pp. 321-341.

PSACHAROPOULOS George, (1988). «Education and Development ». The World Bank Research Observer, Volume 3(1), pp. 99-116.

Rapport du Haut-Commissariat au Plan, (2018). «Les indicateurs sociaux du Maroc », Direction des statistiques, Edition 2018.

RODRIK Dani, (1998). «Why do more open economies have bigger governments? ». Journal of Political Economy 106, pp. 997-1032.

ROGERS Everett, (1983). «Diffusion of Innovations ». 3rd Edition, New York: The Free Press.

ROGERS Everett, (1987). « The diffusion of innovations perspective ». In Neil Weinstein (Ed.), Taking Care: Understanding and Encouraging Self-Protective Behavior Cambridge. Cambridge University Press. pp. 79-94.

ROMER Paul, (1990). «Endogenous Technological Change ». Journal of Political Economy Volume 98, N0 5, Part 2.

SALMI Jamil, (1987). «Language and schooling in Morocco ». International Journal of Educational Development, Volume 7, Issue 1, pp. 21-31.

SCHULTZ Theodore, (1961). «Investment in Human Capital ». The American Economic Review, Volume 51, No. 1, pp. 1-17.

SCHUMPETER Joseph Alois, (1934). « The Theory of Economic Development: An Inquiry into Profits, Capital, Credit, Interest, and the Business Cycle ». Harvard Economic Studies 46.

SEDDON David, (1989). « The Politics of Adjustment in Morocco », Structural Adjustment in Africa. Part of the International Political Economy Series book series (IPES), B. K. Campbell et al. (eds.), pp. 234-265. 
SMITH Adam, (1776). «Wealth of nations », Volume I, Book II, Editions of Cananique, pp. 264.

SOLOW Robert, (1956). «A Contribution to the Theory of Economic Growth ». The Quarterly Journal of Economics, Volume 70, No. 1 (Feb., 1956), The MIT Press, pp. 65-94.

SPENGLER Joseph, (1977). « Adam Smith on Human Capital », The American Economic Review, Volume 67, No. 1, Papers and Proceedings of the Eightyninth Annual Meeting of the American Economic Assocation (February, 1977), pp. 32-36.

TAWIL Sobhi, CERBELLE Sophie, AMAPOLA Alama, (2010). «Education au Maroc: analyse du secteur ». Documents de l'UNESCO N0 48223, Bureau multipays pour le Maghreb, pp. 01-90.

WALSH Robert, (1935). «Capital Concept Applied to Man ». The Quarterly Journal of Economics, Volume 49, No. 2, pp. 255-285.

WEST Edwin George, (1964). «The role of education in nineteenth-century doctrines of political economy ». British Journal of Educational Studies, 12(2), pp. 161172. 